\title{
Motivation Affects Sports and Life Skills in Physical Disabled People
}

\section{La motivación afecta los deportes y las habilidades para la vida en personas con discapacidad física}

\author{
Çağrı ÇELENK \\ Assoc.Prof.Dr. University of Erciyes, Faculty of Sport Sciences, Kayseri, TURKEY \\ ORCID: https://orcid.org/0000-0003-2448-3011
}

Received 09-08-20 Revised 10-10-20

\section{* Correspondence}

Email: celenkc@erciyes.edu.tr
Accepted 12-12-20 On line 03-17-21

\section{Citation:}

Çağrı ÇELENK. (2021). Motivation Affects Sports and Life Skills in Physical Disabled People. Propósitos y Representaciones, 9 (SPE3), e1161. Doi: http://dx.doi.org/10.20511/pyr2021.v9nSPE3.1161 


\begin{abstract}
In this study, it has been tried to determine the motivation of participation in sports and the effects of sports on their lifestyles of different physically disabled individuals who do sports and those who do not, and the differences between the types of disabilities were examined. A total of 732 physically disabled individuals including 327 people who do sports and 405 people who do not do sports were included in the study. Personal Information Form, Physical Activity Participation Motivation Scale (PAPMS) and the Effect of Sports on Life Skills Scale (SLSS) were used as data collection tools There is a statistically significant difference between those who do sports and those who do not in total and all sub-parameters in PAPMS and SLSS $(\mathrm{p}<0.05)$. When the total scores and subdimensions of the participants are examined, there is a positive correlation between PAPMS and SLSS $(\mathrm{p}<0.05)$. When the responses of the participants to FAPMS and SLSS are compared according to the type of disability, there is a statistically significant difference between those who do sports and those who do not in the total and sub-dimensions of Orthopedic, Visually and hearing impaired individuals $(p<0.05)$. As a result, it shows that sports have a positive role in the motivation of participating in physical activities and contribute to life skills on disabled individuals who do sports. It is thought that sports will have a positive effect not only on their participation in physical activities, but also in many cognitive learning processes due to the increase in motivation of people with disabilities and help the disabled person to become more active and accepted in the society.
\end{abstract}

Keywords: Sport, Motivation, Life Skill, Disabled

\title{
Resumen
}

En este estudio se ha intentado determinar la motivación de la participación en el deporte y los efectos del deporte en sus estilos de vida de diferentes individuos con discapacidad física que practican deporte y los que no, y se examinaron las diferencias entre los tipos de discapacidad. . Se incluyeron en el estudio un total de 732 personas con discapacidad física, incluidas 327 personas que practican deportes y 405 personas que no practican deportes. El formulario de información personal, la escala de motivación para la participación en la actividad física (PAPMS) y la escala del efecto de los deportes en las habilidades para la vida (SLSS) se utilizaron como herramientas de recopilación de datos. Existe una diferencia estadísticamente significativa entre los que practican deporte y los que no lo hacen en total y todos los subparámetros en PAPMS y SLSS $(\mathrm{p}<0,05)$. Cuando se examinan las puntuaciones totales y las subdimensiones de los participantes, existe una correlación positiva entre PAPMS y SLSS $(\mathrm{p}<0,05)$. Cuando se comparan las respuestas de los participantes a FAPMS y SLSS según el tipo de discapacidad, existe una diferencia estadísticamente significativa entre los que practican deporte y los que no lo hacen en el total y subdimensiones de Ortopédicos, Discapacitados visuales y auditivos. $(\mathrm{p}<0,05)$. Como resultado, muestra que el deporte tiene un papel positivo en la motivación de participar en actividades físicas y contribuye a las habilidades para la vida de las personas con discapacidad que practican deporte. Se piensa que el deporte tendrá un efecto positivo no solo en su participación en actividades físicas, sino también en muchos procesos de aprendizaje cognitivo debido al aumento de la motivación de las personas con discapacidad y ayudarán a la persona con discapacidad a ser más activa y aceptada en la sociedad. .

Palabras clave: Deporte, Motivación, Habilidad para la vida, Discapacitados

\section{Introduction}

World Health Organization (WHO) stated that the world is more than 1 billion people with disabilities (WHO, 2011), this ratio is approximately 8.5 Turkey (12:29\% of the population) are million. (Die, 2009). People with disabilities generally have worse health conditions than nondisabled people (WHO, 2011). Physical activity (PA) has beneficial effects on health for all individuals (Akil, 2012; Top, Akil \& Aydin, 2018; Top, Kibris \& Kargi, 2020). Despite this, almost half of individuals with disabilities are physically inactive and are likely to have a chronic disease (Carroll et al., 2014). Having PA reduces the risk of chronic disease (Durstine, Gordon, Wang, \& 
Luo, 2013), with muscle strength and functional movement (Çagri, Marangoz, Aktug, Top \& Akil 2015; Akil Et Al., 2016; Top \& Akil, 2018) helps to improve the quality of life (De Hollander, \& Proper, 2018; Kosowan, 2019). Therefore, disabled individuals should be supported to find PA. Because they can be more active when they are supported, and they get discouraged when they fail (Bragaru, Et Al., 2013). Therefore, when they fail, their motivation should be re-activated.

Motivation is highly effective on human behavior and enables individuals to act (Cottingham, Phillips, Hall, Gearity, \& Carroll, 2014). Healthy individuals and individuals with disabilities (Trost, Owen, Bauman, Sallis \& Brown, 2002) need motivation to be active in terms of sports (Deans, Burns, Mcgarry, Murray, \& Mutrie, 2012; Buffart Et Al., 2009). Although motivation has been cited as a necessary factor for maintaining physical activity in persons with disabilities (Skatteboe, et al., 2016), studies have focused more on examining motivation within competitive sports (Chantal, Guay, Dobreva-Martinova \& Vallerand, 1996; Rogers, Morris, \& Moore, 2008). However, first of all, differences in motivation between individuals who do sports and those who do not should be determined and whether they affect their lives should be examined.

Life skills must be developed for individuals to realize their true potential (Bell, \& Quintal, 1985). Because these skills are critical to dealing with life's daily challenges (Rubin, Chan, \& Thomas, 2003; Kingsnorth, Healy, \& Macarthur, 2007). Sport is an effective method for developing skills and transferring these skills to their own lives (Perkins, \& Noam, 2007). Jones, Lavallee, and Tod (2011) showed that individuals learn life skills such as motivation and communication skills through sports. This is important as it shows us that sports contributes to the development of life skills and that motivation is needed to continue doing sports (Lee, Park, Jang, \& Park, 2017; O'neil \& Hodge, 2019).

Although it is shown in studies that sports increase life skills (PERKINS, \& NOAM, 2007) and that the effects of motivation in sports should be taken into account (KAVUSSANU, 2006), there is no such study comparing physically disabled individuals who do and do not do sports. For this reason, our first research question in our study is to what level is the motivation level and life skills of physically disabled individuals who do sports and those who do not, and is there any difference between them. Our second research question is whether there is a correlation between motivation and life skills. 3. Our research question is whether there is a difference between different types of disabilities as doing sports or not, and our research question is to determine whether the types of disability differ between each other in terms of motivation or life skills.

\section{Method}

\section{Study group}

732 people, 517 (70.6\%) male and 215 (29.4\%) female, participated in the study. 327 participants (44.7\%) do licensed sports, while $405(55.3 \%)$ do not. Of the 327 people engaged in sports, 257 (78.6\%) are men and $70(21.4 \%)$ are women. Among the participants doing sports, $114(34.86 \%)$ people were Football, 50 people (15.29\%) Goalball, 42 people $(12.85 \%)$ Basketball, 31 people (9.48\%) Athletics, 30 people $(9.17 \%)$ Table Tennis, 29 people $(8.87 \%)$ Swimming, 31 people (9.41\%) are interested in Wrestling. $260(64.2 \%)$ of the 405 people who do not do sports are men and $145(35.8 \%)$ are women. Among the participants, 272 (37.2\%) are orthopedic, $209(28.6 \%)$ are visually impaired, and $251(34.3 \%)$ are hearing impaired. Of the 327 participants, 101 (30.9\%) are orthopedic, 130 (39.8\%) are visually impaired, 96 (29.4\%) are hearing impaired. Of the 405 people who do not do sports, $171(42.2 \%)$ are orthopedic, $79(19.5 \%)$ are blind, $155(38.3 \%)$ are hearing impaired. Of the 327 people who did sports, 220 (67.3\%) were congenital disabilities and 107 (32.7\%) were disabled afterwards. Of the 405 people who do not do sports, 257 (63.5\%) became disabled from birth, $148(36.5 \%)$ later became disabled. The surveys were applied to 500 people who do active sports and 500 physically disabled individuals who do not do sports. However, 327 questionnaires of disabled individuals who do active sports and 405 questionnaires of disabled individuals who do not do active sports were evaluated. The scales were administered using face-toface interview technique by expert interviewers trained in this field. Before filling the relevant forms, the participants were informed about the study and their verbal consent was obtained. 


\section{Data Collection Tools}

\section{Physical Activity Participation Motivation Scale (PAPMS)}

The scale developed by Tekkurşun \& Cicioğlu (2018) consists of 16 items and 3 sub-dimensions. Sub-dimensions Individual Causes (Items 1,2,3,4,5,6), Environmental Causes (Items 8,9,10,11,12) and Cause (Items 7,13,14,15,16) expressed as. Some items are reverse coded $(3,9,13,14,15,16)$. The minimum score that can be obtained from the scale is 16 and the maximum score is 80 . The scores indicate that they have 1-16 very low, 17-32 low, 33-48 medium, 49- 64 high, 65-80 very high motivation to participate in physical activity. The Cronbach Alpha values of the scale are between .82 and .89. In our study, the Cronbach $\alpha$ internal consistency value of the Scale of Motivation for Participation in Physical Activity for Persons with Disabilities, which consists of 16 items, was 0.844 . The scale is a valid and reliable measurement tool.

\section{The Scale of the Effects of Sports on Life Skills (SLS)}

The scale was developed by Cronin and Allen (2017). It was adapted to Turkish by Düz and Açak (2018). The scale consists of 31 questions and 7 sub-dimensions. Sub-dimensions "Time management (1,2,3,4. Questions)", "Communication (5,6,7,8. Questions)", "Leadership (9,10,11,12,13. Questions)", "Teamwork (14,15,16,17,18,19. Questions)", "Social skills $(20,21,22,23$. Questions)", "Emotional skills (24,25,26,27. Questions) "And" Setting a goal $(28,29,30,31$ questions) ". The scale is scored on a 5-point Likert scale as "I do not agree at all (1)" and "I completely agree (5)". There is no reverse scored expression in the scale. The lowest score that can be obtained from the scale is 31 , and the highest score is 155 . Within the scope of the reliability study, the internal consistency coefficient was found to be .92, and the Pearson Product Moment Correlation coefficient $(\mathrm{r}=.89)$ calculated for the test-retest reliability study was found to be high and significant. In our study, the Cronbach $\alpha$ internal consistency value of HDSS, which consists of 31 items, was examined and the value was determined as 0.959 . The scale is a valid and reliable measurement tool.

\section{Statistical Analysis}

SPSS 21.0 package program was used in the analysis of the study data. Descriptive statistics were used as number, percentage, mean and standard deviation. In order to determine whether parametric analysis will be performed or not, it was examined whether the data showed normal distribution. Independent groups $\mathrm{T}$ test was used for paired comparisons with normal distribution FACMS and HDSS. Multiple comparisons were evaluated with One-Way Anova and the second level test with Tukey. Statistical significance level was accepted as $\mathrm{p}<0.05$.

\section{Findings}

Table 1. Comparison of PAPM and SLS levels according to doing and not doing sports

\begin{tabular}{|c|c|c|c|c|c|c|c|}
\hline Dimension & An athlete? & $\mathbf{N}$ & Min & Max & $\square$ & $\mathbf{t}$ & p \\
\hline \multirow{2}{*}{ Individual Causes } & Yes & 327 & 6 & 30 & $24.18 \pm 4.19$ & \multirow{2}{*}{10.907} & \multirow{2}{*}{$0.000 *$} \\
\hline & No & 405 & 6 & 30 & $18.96 \pm 7.80$ & & \\
\hline \multirow{2}{*}{ Environmental Causes } & Yes & 327 & 5 & 25 & $20.69 \pm 4.36$ & \multirow{2}{*}{8.114} & \multirow{2}{*}{$0.000 *$} \\
\hline & No & 405 & 5 & 25 & $16.94 \pm 7.37$ & & \\
\hline \multirow{2}{*}{ Causelessness } & Yes & 327 & 5 & 25 & $15.80 \pm 4.66$ & \multirow{2}{*}{6.986} & \multirow{2}{*}{$0.000 *$} \\
\hline & No & 405 & 5 & 25 & $13.06 \pm 5.71$ & & \\
\hline \multirow{2}{*}{ PAPM (Total) } & Yes & 327 & 16 & 80 & $60.66 \pm 9.41$ & \multirow{2}{*}{10.262} & \multirow{2}{*}{$0.000 *$} \\
\hline & No & 405 & 16 & 80 & $48.96 \pm 18.81$ & & \\
\hline \multirow{2}{*}{ Time management } & Yes & 327 & 4 & 20 & $15.42 \pm 3.09$ & \multirow{2}{*}{12.726} & \multirow{2}{*}{$0.000 *$} \\
\hline & No & 405 & 4 & 20 & $10.93 \pm 5.75$ & & \\
\hline \multirow[t]{3}{*}{ Contact } & Yes & 327 & 4 & 20 & $15.20 \pm 3.01$ & \multirow{3}{*}{11.386} & \multirow{3}{*}{$0.000 *$} \\
\hline & No & 405 & 4 & 20 & $11.15 \pm 5.85$ & & \\
\hline & Yes & 327 & 5 & 25 & $19.06 \pm 3.45$ & & \\
\hline
\end{tabular}




\begin{tabular}{|c|c|c|c|c|c|c|c|}
\hline Leadership & No & 405 & 5 & 25 & $13.63 \pm 6.97$ & 12.865 & $0.000 *$ \\
\hline \multirow{2}{*}{ Team work } & Yes & 327 & 6 & 30 & $23.66 \pm 4.00$ & \multirow{2}{*}{12.590} & \multirow{2}{*}{$0.000 *$} \\
\hline & No & 405 & 6 & 30 & $17.24 \pm 8.48$ & & \\
\hline \multirow{2}{*}{ Social Skills } & Yes & 327 & 4 & 20 & $15.81 \pm 2.76$ & \multirow{2}{*}{12.175} & \multirow{2}{*}{$0.000 *$} \\
\hline & No & 405 & 4 & 20 & $11.48 \pm 5.93$ & & \\
\hline \multirow{2}{*}{ Emotional Skills } & Yes & 327 & 4 & 20 & $15.30 \pm 2.88$ & \multirow{2}{*}{12.959} & \multirow{2}{*}{$0.000 *$} \\
\hline & No & 405 & 4 & 20 & $10.86 \pm 5.62$ & & \\
\hline \multirow{2}{*}{ Setting a goal } & Yes & 327 & 4 & 20 & $15.39 \pm 3.62$ & \multirow{2}{*}{11.273} & \multirow{2}{*}{$0.000 *$} \\
\hline & No & 405 & 4 & 20 & $11.17 \pm 5.95$ & & \\
\hline \multirow{2}{*}{ SLS (Total) } & Yes & 327 & 31 & 155 & $119.85 \pm 17.48$ & \multirow{2}{*}{13.612} & \multirow[t]{2}{*}{$0.000 *$} \\
\hline & No & 405 & 31 & 155 & $86.46 \pm 41.47$ & & \\
\hline
\end{tabular}

* Significant difference at $\mathrm{p}<0.05$ level. PAPM: Physical Activity Participation Motivation Scale;

SLS: The Effects of Sports on Life Skills

When the answers given by the participants to PAPM are examined, those who do sports have obtained $=60.66 \pm 9.41$ points in total, and those who do not do sports have obtained $=48.96$ \pm 18.81 points. When their responses to PAPMS are compared, there is a statistically significant difference between those who do sports and those who do not in total and all sub-parameters ( $\mathrm{p}$ $<0.05$ ). When the answers given by the participants to the SLSS were examined, those who did sports obtained $=119.85 \pm 17.48$ points, and those who did not do sports obtained $=86.46 \pm 41.47$ points. When the responses of the participants to the SLSS are compared, there is a statistically significant difference between those who do sports and those who do not in total and all subparameters $(\mathrm{p}<0.05$; Table 1$)$.

Table 2. Comparison of Disability Type PAPM levels according to Sports Practice

\begin{tabular}{|c|c|c|c|c|c|c|}
\hline & Type of Disability & An athlete? & $\mathbf{N}$ & $\mathbf{M E A N} \pm \mathbf{S D}$ & $\mathbf{t}$ & $\mathbf{P}$ \\
\hline \multirow{6}{*}{ Individual Causes } & \multirow{2}{*}{ Orthopedic } & Yes & 101 & $24.15 \pm 3.92$ & \multirow{2}{*}{4.683} & \multirow{2}{*}{0.000} \\
\hline & & No & 171 & $19.98 \pm 8.43$ & & \\
\hline & \multirow{2}{*}{ Visually } & Yes & 130 & $25.45 \pm 3.72$ & \multirow{2}{*}{6.977} & \multirow{2}{*}{0.000} \\
\hline & & No & 79 & $19.71 \pm 8.08$ & & \\
\hline & \multirow{2}{*}{ Hearing } & Yes & 96 & $22.51 \pm 4.49$ & \multirow{2}{*}{6.559} & \multirow{2}{*}{0.000} \\
\hline & & No & 155 & $17.45 \pm 6.68$ & & \\
\hline \multirow{6}{*}{$\begin{array}{l}\text { Environmental } \\
\text { Causes }\end{array}$} & \multirow{2}{*}{ Orthopedic } & Yes & 101 & $20.63 \pm 4.33$ & \multirow{2}{*}{4.328} & \multirow{2}{*}{0.000} \\
\hline & & No & 171 & $16.94 \pm 7.90$ & & \\
\hline & \multirow{2}{*}{ Visually } & Yes & 130 & $20.54 \pm 4.61$ & \multirow{2}{*}{4.254} & \multirow{2}{*}{0.000} \\
\hline & & No & 79 & $16.92 \pm 7.68$ & & \\
\hline & \multirow{2}{*}{ Hearing } & Yes & 96 & $20.94 \pm 4.07$ & \multirow{2}{*}{5.323} & \multirow{2}{*}{0.000} \\
\hline & & No & 155 & $16.95 \pm 6.61$ & & \\
\hline \multirow{6}{*}{ Causelessness } & \multirow{2}{*}{ Orthopedic } & Yes & 101 & $16.00 \pm 4.77$ & \multirow{2}{*}{3.450} & \multirow{2}{*}{0.001} \\
\hline & & No & 171 & $13.63 \pm 5.86$ & & \\
\hline & \multirow{2}{*}{ Visually } & Yes & 130 & $16.85 \pm 3.61$ & \multirow{2}{*}{4.906} & \multirow{2}{*}{0.000} \\
\hline & & No & 79 & $13.51 \pm 6.23$ & & \\
\hline & \multirow{2}{*}{ Hearing } & Yes & 96 & $14.16 \pm 5.34$ & \multirow{2}{*}{2.866} & \multirow{2}{*}{0.005} \\
\hline & & No & 155 & $12.21 \pm 5.17$ & & \\
\hline \multirow{6}{*}{ PAPM (Total) } & \multirow{2}{*}{ Orthopedic } & Yes & 101 & $60.78 \pm 9.33$ & \multirow{2}{*}{4.721} & 0.000 \\
\hline & & No & 171 & $50.54 \pm 20.57$ & & 0.000 \\
\hline & Visually & Yes & 130 & $62.83 \pm 8.43$ & 6338 & 0000 \\
\hline & visuany & No & 79 & $50.14 \pm 20.14$ & 0.500 & 0.000 \\
\hline & Hearing & Yes & $\frac{96}{155}$ & $57.60 \pm 10.01$ & 6.130 & 0.000 \\
\hline & & No & 155 & $46.61 \pm 15.71$ & & \\
\hline
\end{tabular}

* Significant difference at $\mathrm{p}<0.05$ level. PAPM: Physical Activity Participation Motivation Scale; SLS: The Effects of Sports on Life Skills

When the answers given by the participants to PAPM, the difference levels within the type of disability are compared, there is a statistically significant difference between the individuals who do sports in the total and sub-dimensions of orthopedic, visually and hearing impaired individuals and those who do not $(\mathrm{p}<0.05)$.

Table 3. Comparison of Disability Type and SLS levels by Sport Status

\begin{tabular}{lllllll}
\hline Type of Disability & An & N & MEAN \pm SD & t & P \\
\hline
\end{tabular}




\begin{tabular}{|c|c|c|c|c|c|}
\hline & & thlete & & & \\
\hline \multirow{6}{*}{ Time management } & \multirow{2}{*}{ Orthopedic } & Yes & 101 & $16.05 \pm 2.88$ & \multirow{2}{*}{0.000} \\
\hline & & No & 171 & $11.37 \pm 6.17$ & \\
\hline & \multirow{2}{*}{ Visually } & Yes & 130 & $15.60 \pm 3.07$ & \multirow{2}{*}{0.000} \\
\hline & & No & 79 & $10.20 \pm 5.71$ & \\
\hline & \multirow{2}{*}{ Hearing } & Yes & 96 & $14.52 \pm 3.15$ & \multirow{2}{*}{0.000} \\
\hline & & No & 155 & $10.82 \pm 5.25$ & \\
\hline \multirow{6}{*}{ Contact } & \multirow{2}{*}{ Orthopedic } & Yes & 101 & $16.12 \pm 2.47$ & \multirow{2}{*}{0.000} \\
\hline & & No & 171 & $11.82 \pm 6.24$ & \\
\hline & \multirow{2}{*}{ Visually } & Yes & 130 & $15.24 \pm 2.62$ & \multirow{2}{*}{0,000} \\
\hline & & No & 79 & $10.56 \pm 6.27$ & \\
\hline & \multirow{2}{*}{ Hearing } & Yes & 96 & $14.20 \pm 3.67$ & \multirow{2}{*}{0.000} \\
\hline & & No & 155 & $10.70 \pm 5.10$ & \\
\hline \multirow{6}{*}{ Leadership } & \multirow{2}{*}{ Orthopedic } & Yes & 101 & $19.58 \pm 3.36$ & 0000 \\
\hline & & No & 171 & $14.18 \pm 7.36$ & 0.000 \\
\hline & Visually & Yes & 130 & $19.13 \pm 3.16$ & 0000 \\
\hline & visuany & No & 79 & $12.73 \pm 7.17$ & 0.000 \\
\hline & Hearing & Yes & 96 & $18.42 \pm 3.83$ & 0000 \\
\hline & Hearing & No & 155 & $13.50 \pm 6.39$ & 0.000 \\
\hline & Orthonedic & Yes & 101 & $24.22 \pm 3.15$ & 0000 \\
\hline & Ortnopedic & No & 171 & $17.66 \pm 8.87$ & 0.000 \\
\hline Team work & Visually & Yes & 130 & $24.17 \pm 3.97$ & 0000 \\
\hline & visuany & No & 79 & $16.44 \pm 9.27$ & 0.000 \\
\hline & & Yes & 96 & $22.38 \pm 4.55$ & \\
\hline & Hearıng & No & 155 & $17.19 \pm 7.61$ & 0.000 \\
\hline & Orthopedic & Yes & 101 & $15.96 \pm 2.60$ & 0.000 \\
\hline & & No & 171 & $12.13 \pm 6.31$ & 0.000 \\
\hline Social Skills & Visually & Yes & 130 & $16.44 \pm 2.73$ & 0000 \\
\hline & & No & 79 & $10.87 \pm 6.26$ & 0.000 \\
\hline & & Yes & 96 & $14.80 \pm 2.70$ & \\
\hline & Hearıng & No & 155 & $11.07 \pm 5.26$ & 0.000 \\
\hline & Orthonedic & Yes & 101 & $15.50 \pm 2.68$ & 0.000 \\
\hline & & No & 171 & $11.08 \pm 5.89$ & \\
\hline Emotional Skills & Visually & Yes & 130 & $15.48 \pm 2.85$ & 0000 \\
\hline Emotional SKills & visuany & No & 79 & $10.85 \pm 6.04$ & 0.000 \\
\hline & Hearing & Yes & 96 & $14.84 \pm 3.10$ & 0000 \\
\hline & Hearming & No & 155 & $10.62 \pm 5.11$ & 0.000 \\
\hline & Orthonedic & Yes & 101 & $15.66 \pm 3.91$ & 0000 \\
\hline & & No & 171 & $11.32 \pm 6.09$ & \\
\hline Setting a goal & Visually & Yes & 130 & $15.49 \pm 3.77$ & 0000 \\
\hline & visuany & No & 79 & $10.32 \pm 6.28$ & 0.000 \\
\hline & Hearing & Yes & 96 & $14.98 \pm 3.05$ & 0.000 \\
\hline & nearmg & No & 155 & $11.43 \pm 5.62$ & \\
\hline & Orthonedic & Yes & 101 & $123.09 \pm 15.087360$ & 0000 \\
\hline & Orinopedic & No & 171 & $89.56 \pm 44.27$ & 0.000 \\
\hline SLS (Total) & Visually & Yes & 130 & $121.55 \pm 16.48$ & 0000 \\
\hline SLS (10tal) & & No & 79 & $81.97 \pm 44.43 \quad 9.181$ & 0.000 \\
\hline & Hearing & Yes & 96 & $114.14 \pm 19.82 \quad 7119$ & 0000 \\
\hline & Hearming & No & 155 & $85.34 \pm 36.42$ & 0.000 \\
\hline
\end{tabular}

* Significant difference at $\mathrm{p}<0.05$ level. PAPM: Physical Activity Participation Motivation Scale; SLS: The Effects of Sports on Life Skills

When the responses of the participants to the SLS are compared, there is a statistically significant difference between those who do sports and those who do not, in total and sub-dimensions of orthopedic, visually and hearing impaired individuals $(\mathrm{p}<0.05)$.

Table 4. Comparison of PAPM and SLS levels for those doing sports according to the type of disability

\begin{tabular}{cccccc}
\hline & $\begin{array}{c}\text { Type of } \\
\text { Disability }\end{array}$ & N & MEAN \pm SD & F & P \\
\hline Individual Causes & Orthopedic & 101 & $24.15 \pm 3.92$ b & 14.711 & $0.000 *$ \\
\hline & & & & $\begin{array}{r}\text { Propósitos y Representaciones } \\
\text { May. 2021, Vol. } 9 \text {, SPE(3), en161 }\end{array}$ \\
& & & http://dx.doi.org/10.20511/pyr2021.v9nSPE3.1161
\end{tabular}




\begin{tabular}{|c|c|c|c|c|c|}
\hline & Visually & 130 & $25.45 \pm 3.72^{a}$ & & \\
\hline & Hearing & 96 & $22.51 \pm 4.49^{\mathrm{c}}$ & & \\
\hline \multirow{3}{*}{ Environmental Causes } & Orthopedic & 101 & $20.63 \pm 4.33$ & \multirow{3}{*}{0.240} & \multirow{3}{*}{0.787} \\
\hline & Visually & 130 & $20.54 \pm 4.61$ & & \\
\hline & Hearing & 96 & $20.94 \pm 4.07$ & & \\
\hline \multirow{3}{*}{ Causelessness } & Orthopedic & 101 & $16.00 \pm 4.77^{\mathrm{a}}$ & \multirow{3}{*}{9.857} & \multirow{3}{*}{$0.000 *$} \\
\hline & Visually & 130 & $16.85 \pm 3.61^{\mathrm{a}}$ & & \\
\hline & Hearing & 96 & $14.16 \pm 5.34^{b}$ & & \\
\hline \multirow{3}{*}{ PAPM (Total) } & Orthopedic & 101 & $60.78 \pm 9.33^{\mathrm{a}}$ & \multirow{3}{*}{8.941} & \multirow{3}{*}{$0.000 *$} \\
\hline & Visually & 130 & $62.83 \pm 8.43^{\mathrm{a}}$ & & \\
\hline & Hearing & 96 & $57.60 \pm 10.01^{\mathbf{b}}$ & & \\
\hline \multirow{3}{*}{ Time management } & Orthopedic & 101 & $16.05 \pm 2.88^{\mathrm{a}}$ & \multirow{3}{*}{6.615} & \multirow{3}{*}{$0.002 *$} \\
\hline & Visually & 130 & $15.60 \pm 3.07^{\mathrm{a}}$ & & \\
\hline & Hearing & 96 & $14.52 \pm 3.15^{b}$ & & \\
\hline \multirow{3}{*}{ Contact } & Orthopedic & 101 & $16.12 \pm 2.47^{\mathrm{a}}$ & \multirow{3}{*}{10.607} & \multirow{3}{*}{$0.000 *$} \\
\hline & Visually & 130 & $15.24 \pm 2.62^{\mathrm{a}}$ & & \\
\hline & Hearing & 96 & $14.20 \pm 3.67^{\mathbf{b}}$ & & \\
\hline \multirow{3}{*}{ Leadership } & Orthopedic & 101 & $19.58 \pm 3.36$ & \multirow{3}{*}{2.896} & \multirow{3}{*}{0.057} \\
\hline & Visually & 130 & $19.13 \pm 3.16$ & & \\
\hline & Hearing & 96 & $18.42 \pm 3.83$ & & \\
\hline \multirow{3}{*}{ Team work } & Orthopedic & 101 & $24.22 \pm 3.15^{\mathrm{a}}$ & \multirow{3}{*}{7.273} & \multirow{3}{*}{$0.001 *$} \\
\hline & Visually & 130 & $24.17 \pm 3.97^{\mathrm{a}}$ & & \\
\hline & Hearing & 96 & $22.38 \pm 4.55^{\mathbf{b}}$ & & \\
\hline \multirow{3}{*}{ Social Skills } & Orthopedic & 101 & $15.96 \pm 2.60^{\mathrm{a}}$ & \multirow{3}{*}{10.514} & \multirow{3}{*}{$0.000 *$} \\
\hline & Visually & 130 & $16.44 \pm 2.73^{\mathrm{a}}$ & & \\
\hline & Hearing & 96 & $14.80 \pm 2.70^{b}$ & & \\
\hline \multirow{3}{*}{ Emotional Skills } & Orthopedic & 101 & $15.50 \pm 2.68$ & \multirow{3}{*}{1.687} & \multirow{3}{*}{0.187} \\
\hline & Visually & 130 & $15.48 \pm 2.85$ & & \\
\hline & Hearing & 96 & $14.84 \pm 3.10$ & & \\
\hline \multirow{3}{*}{ Setting a goal } & Orthopedic & 101 & $15.66 \pm 3.91$ & \multirow{3}{*}{0.958} & \\
\hline & Visually & 130 & $15.49 \pm 3.77$ & & 0.385 \\
\hline & Hearing & 96 & $14.98 \pm 3.05$ & & \\
\hline & Orthopedic & 101 & $123.09 \pm 15.08^{\mathrm{a}}$ & & \\
\hline SLS (Total) & Visually & 130 & $121.55 \pm 16.48^{a}$ & 7.783 & $0.000 *$ \\
\hline & Hearing & 96 & $114.14 \pm 19.82^{b}$ & & \\
\hline
\end{tabular}

* Significant difference at $\mathrm{p}<0.05$ level.. $\quad$ PAPM: Physical Activity Participation Motivation Scale; SLS: The Effects of Sports on Life Skills a, b, c: Different letters represent the difference between groups.

When the responses of the participants who do sports to PAPM and SLS are compared according to the type of disability, Individual Causes, Cause, PAPM (Total), Time Management, Communication, Teamwork, Social Skills, SLS (Total) dimensions Orthopedic, Visually and hearing impaired individuals There is a statistically significant difference between $(\mathrm{p}<0.05)$. There is no statistically significant difference in environmental reasons, leadership, emotional skills, and goal setting sub-dimensions $(\mathrm{p}>0.05)$.

Table 5. Comparison of PAPM and SLS levels for those who do not do sports according to the type of disability

\begin{tabular}{|c|c|c|c|c|c|}
\hline & Type of Disability & $\mathbf{N}$ & MEAN \pm SD & $\mathbf{F}$ & $\mathbf{P}$ \\
\hline \multirow{3}{*}{ Individual Causes } & Orthopedic & 171 & $19.98 \pm 8.43^{\mathrm{a}}$ & \multirow{3}{*}{4.800} & \multirow{3}{*}{$0.009 *$} \\
\hline & Visually & 79 & $19.71 \pm 8.08^{\mathbf{a b}}$ & & \\
\hline & Hearing & 155 & $17.45 \pm 6.68^{\mathrm{b}}$ & & \\
\hline \multirow{3}{*}{ Environmental Causes } & Orthopedic & 171 & $16.94 \pm 7.90$ & \multirow{3}{*}{0.000} & \multirow{3}{*}{1.000} \\
\hline & Visually & 79 & $16.92 \pm 7.68$ & & \\
\hline & Hearing & 155 & $16.95 \pm 6.61$ & & \\
\hline
\end{tabular}




\begin{tabular}{|c|c|c|c|c|c|}
\hline \multirow{3}{*}{ Causelessness } & Orthopedic & 171 & $13.63 \pm 5.86$ & \multirow{3}{*}{2.836} & \multirow{3}{*}{0.060} \\
\hline & Visually & 79 & $13.51 \pm 6.23$ & & \\
\hline & Hearing & 155 & $12.21 \pm 5.17$ & & \\
\hline \multirow{3}{*}{ PAPM (Total) } & Orthopedic & 171 & $50.54 \pm 20.57$ & \multirow{3}{*}{1.984} & \multirow{3}{*}{0.139} \\
\hline & Visually & 79 & $50.14 \pm 20.14$ & & \\
\hline & Hearing & 155 & $46.61 \pm 15.71$ & & \\
\hline \multirow{3}{*}{ Time management } & Orthopedic & 171 & $11.37 \pm 6.17$ & \multirow{3}{*}{1.160} & \multirow{3}{*}{0.315} \\
\hline & Visually & 79 & $10.20 \pm 5.71$ & & \\
\hline & Hearing & 155 & $10.82 \pm 5.25$ & & \\
\hline \multirow{3}{*}{ Contact } & Orthopedic & 171 & $11.82 \pm 6.24$ & \multirow{3}{*}{1.985} & \multirow{3}{*}{0.139} \\
\hline & Visually & 79 & $10.56 \pm 6.27$ & & \\
\hline & Hearing & 155 & $10.70 \pm 5.10$ & & \\
\hline \multirow{3}{*}{ Leadership } & Orthopedic & 171 & $14.18 \pm 7.36$ & \multirow{3}{*}{1.206} & \multirow{3}{*}{0.301} \\
\hline & Visually & 79 & $12.73 \pm 7.17$ & & \\
\hline & Hearing & 155 & $13.50 \pm 6.39$ & & \\
\hline \multirow{3}{*}{ Team work } & Orthopedic & 171 & $17.66 \pm 8.87$ & \multirow{3}{*}{0.560} & \multirow{3}{*}{0.571} \\
\hline & Visually & 79 & $16.44 \pm 9.27$ & & \\
\hline & Hearing & 155 & $17.19 \pm 7.61$ & & \\
\hline \multirow{3}{*}{ Social Skills } & Orthopedic & 171 & $12.13 \pm 6.31$ & \multirow{3}{*}{1.830} & \multirow{3}{*}{0.162} \\
\hline & Visually & 79 & $10.87 \pm 6.26$ & & \\
\hline & Hearing & 155 & $11.07 \pm 5.26$ & & \\
\hline \multirow{3}{*}{ Emotional Skills } & Orthopedic & 171 & $11.08 \pm 5.89$ & \multirow{3}{*}{0.274} & \multirow{3}{*}{0.760} \\
\hline & Visually & 79 & $10.85 \pm 6.04$ & & \\
\hline & Hearing & 155 & $10.62 \pm 5.11$ & & \\
\hline \multirow{3}{*}{ Setting a goal } & Orthopedic & 171 & $11.32 \pm 6.09$ & \multirow{3}{*}{1.014} & \multirow{3}{*}{0.364} \\
\hline & Visually & 79 & $10.32 \pm 6.28$ & & \\
\hline & Hearing & 155 & $11.43 \pm 5.62$ & & \\
\hline \multirow{3}{*}{ SLS (Total) } & Orthopedic & 171 & $89.56 \pm 44.27$ & \multirow{3}{*}{0.995} & \multirow{3}{*}{0.370} \\
\hline & Visually & 79 & $81.97 \pm 44.43$ & & \\
\hline & Hearing & 155 & $85.34 \pm 36.42$ & & \\
\hline
\end{tabular}

* Significant difference at $\mathrm{p}<0.05$ level.. $\quad$ PAPM: Physical Activity Participation Motivation Scale; SLS: The Effects of Sports on Life Skills a, b, c: Different letters represent the difference between groups.

When the responses of the participants who do not do sports to PAPM and SLS are compared according to the type of disability, there is a statistically significant difference between Orthopedic and hearing impaired individuals only in the Individual Causes dimension $(\mathrm{p}<0.05)$. There is no statistically significant difference between orthopedic, visually and hearing impaired individuals in terms of environmental reasons, lack of reason, PAPMS (Total), Time Management, Communication, Leadership, Teamwork, Social Skills, Emotional Skills, Goal Setting and SLS(Total).

\section{Discussion and Conclusion}

Today, it is seen that disability is a situation that can happen to every person, and it can no longer be ignored. Many studies have been done and are being carried out to integrate disabled individuals into society and to eliminate discrimination.

In this study, the motivation of people with disabilities to participate in physical activity and the effect of sports on life skills were examined. When the answers given by the participants to PAPS are examined, those who do sports have obtained high scores from those who do not do sports and there is a statistically significant difference between those who do sports and those who do not in total and all sub-parameters. When the responses of the participants to PAPM are compared, there is a statistically significant difference between the individuals who do sports in the total and subdimensions of orthopedic, visually and hearing impaired individuals and those who do not. When the participants who do not do sports are compared to PAPM and their answers according to the type 
of disability, there is a statistically significant difference between Orthopedic and hearing impaired individuals only in the Individual Reasons dimension $(\mathrm{p}<0.05)$. In the study conducted by Ulukan (2020) on secondary school students, it was observed that students who do sports have higher motivation to participate in physical activity than students who do not do sports, according to their average scores. In addition, according to the average scores of the Individual and Environmental Causes sub-dimensions of the motivation to participate in physical activity scale, it was observed that the students who do sports get higher scores than the students who do not do sports. According to the results of the research conducted by Moreno and Cervello (2005) in order to examine the effects of physical activity levels and gender on the physical self-perception of Spanish adults; It has been observed that sports competence, body consciousness, physical condition and physical endurance of men doing sports are at a higher level than women who do sports and those who do not. In addition, it has been observed that the sports competence, physical condition and endurance of women who do sports are higher than women who do not do sports. According to the analysis results obtained in Özdinç (2005) 's study to determine the opinions of teacher candidates on the relationship between sports and sports participation with socialization; The rate of participation in sports $(51.0 \%)$ of the teacher candidates who stated that there was no one doing sports among their family members showed that they were more $(49.0 \%)$ than the teacher candidates who stated that there was an individual doing sports in their family. Özdinç states that there is a person who does sports among family members as a factor that encourages the individual to participate in sports. However, even if there is no individual who does sports in the family, the person tends to participate in sports activities. Edwards et al. (2005) 's different physicalThe physical self-perception and psychological well-being of people who are regularly involved in activities, exercise and sports and who are not involved in any sports. Similar results were found in his research for comparison. According to the results of this study, a significant difference was found between individuals who are interested in different physical activities, exercise and sports in terms of mental health, psychological well-being and physical self-perception compared to those who are not interested in sports. Individuals with sports habits are more likely to participate in physical activities, which is thought to be due to the socialization characteristic of sports, which is a feature developed by the individual. Because the studies in the literature; Özdinç (2005) made an application involving university students in his study in which he investigated the interaction of sports and social participation. According to the results obtained in the study, the students accepted that sports is a means of socialization. The students participating in the study also gave answers showing that sports is a tool that provides social integration within and between societies. The results of these studies in the literature coincide with the results of our research.

When the scores obtained by the participants from the answers given to the SLS are compared, it is seen that the disabled individuals who do sports get higher scores from the scale subdimension and total scores than those who do not do sports. When the responses of the participants to the SLS are compared, there is a statistically significant difference between those who do sports and those who do not, in total and sub-dimensions of orthopedic, visually and hearing impaired individuals $(p<0.05)$. When the responses of the participants who do sports to SLS are compared according to the type of disability, there is a statistically significant difference between Orthopedic, Visually and hearing impaired individuals in the dimensions of Time Management, Communication, Teamwork, Social Skills, SLS (Total) $(p<0.05)$. There is no statistically significant difference in the sub-dimensions of Leadership, Emotional Skills and Determining a goal $(\mathrm{p}>0.05)$. Comparing the responses of participants who do not do sports to SLS according to the type of disability, there is a statistically significant difference between Orthopedic, Visually and Hearing impaired individuals in the dimensions of Time Management, Communication, Leadership, Teamwork, Social Skills, Emotional Skills, Objective Determination and SLS (Total). When the study is evaluated in general, it is seen that the effect of individuals doing sports on life skills is greater. When the literature is examined, there is no study on the subject. However, considering the effectiveness of sports in our research, it is an undeniable fact that sports has an effect on life skills, as in the name of the scale, naturally, individuals who do sports have better these skills. When the relationship between sports and life skills is examined in the literature; The competitive and dynamic nature of the current period makes it a necessity to have life skills as it brings with it the constant change of living conditions. Sport is considered as one of the main tools for the acquisition of these skills (AÇAK \& DÜZ, 2018). An individual who plays sports learns to respect the rights of others. Social competence skills are 
another area that develops through sports. It becomes stronger in tackling the problems encountered with sports. Life skills such as cooperation and teamwork develop through sports (CANLI \& GÜNAY, 2016). The effects of sports, which is a popular activity field, on life skills include the following areas (AÇAK \& DÜZ, 2018);

It creates an ideal environment for the development of sports life skills. Sports is one of the ways to improve life skills with extracurricular activities. With sports, individuals develop emotionally and socially. Thanks to sports, the mental and physical development of young individuals is progressing positively. Sport provides a positive attitude towards life. Sports create a positive environment while improving the life skills of individuals. Sports contributes to the healthy development of young people by keeping them away from bad habits. As can be seen, sports contribute to the development of life skills by making its presence felt in different areas of human life. It is expected that people will show social development in order to be sensitive to the difficulties of life and to struggle against difficulties (KÜÇÜK \& KOÇ, 2004). The presence of sports in human life is capable of contributing to social development and improving life skills in a positive way. Sport has an important role in socialization of the individual, as it is a social activity that enables the individual to participate in dynamic social environments. Considering that sports is mostly a collective activity in modern societies, individuals who are interested in sports enter into social relations with different groups of people through sportive activities. Sport enables the individual to get rid of his own narrow world and to communicate with other people, beliefs and thoughts in other environments, to be influenced by them and to affect them. In this respect, it can be said that sports support the establishment of new friendships, reinforcement and social cohesion, and plays an important role in reintegrating individuals with disabilities into society (YETIM, 2005).

As a result, it shows that sports have a positive role in the motivation of participating in physical activities and contribute to life skills on disabled individuals who do sports. It is thought that sports will have a positive effect not only on their participation in physical activities, but also in many cognitive learning processes due to the increase in motivation of people with disabilities and help the disabled person to become more active and accepted in the society.

\section{Suggestions}

- By applying it to different disability groups, it can be compared with the results of our study and its linearity can be achieved and a reference can be found in the literature.

- Disabled and healthy individuals can be informed about the effect of sports and they can participate in physical activities regularly.

- Individuals or institutions dealing with the education of persons with disabilities should add sports activities to their curriculum or practices.

\section{References}

Açak, M., \& Düz, S. (2018). Sporun yaşam becerilerine etkisi ölçeğinin Türk kültürüne uyarlanmas1. İnönü Üniversitesi Eğitim Fakültesi Dergisi, 5 (9): 1-13

Açak, M., \& Düz, S. (2018). Sporun Yaşam Becerilerine Etkisi Ölçeğinin Türk Kültürüne Uyarlanmas1/ Adaptation Of Life Skills Scale For Sport into the Turkish Culture. İnönü Üniversitesi Eğitim Bilimleri Enstitüsü Dergisi/ Inonu University Journal of the Graduate School of Education, 5 (9): 74-86.

Akil M, Çelenk Ç, Aktuğ Zb, Marangoz İ, Yilmaz T, Top E. (2016). The effect of lower extremity masses and volumes on the balance performance of athletes. Biomedical Research, 27(3), 877-882.

Akil M. (2012). Effect of acute exercises applied to sedentaries on various enzyme levels related to muscle damages. African Journal of Microbiology Research, 6 (2): 284-287.

Bell, C., \& Quintal, J. (1985). A life skills program for physically disabled adolescents. Canadian journal of occupational therapy. Revue canadienne d'ergotherapie, 52(5), 235-239.

Bragaru, M., Van Wilgen, C. P., Geertzen, J. H., Ruijs, S. G., Dijkstra, P. U., \& Dekker, R. (2013). Barriers and facilitators of participation in sports: a qualitative study on Dutch individuals 
with lower limb amputation. PloS one, 8(3), 59881.

Çagri, Ç., Marangoz, I., Aktug, Z.B., Top, E., \& Akil, M. (2015). The effect of quadriceps femoris and hamstring muscular force on static and dynamic balance performance. International Journal of Physical Education, Sports and Health, 2(2), 323-325.

Canli, U., \& Günay, M. (2016). Basketbol oynayan erkek çocukların akademik başarılarının bazı değişkenler ile ilişkisi. Uluslararası Spor Egzersiz ve Antrenman Bilimi Dergisi, 2 (2): 39 49.

Carroll, D. D., Courtney-Long, E. A., Stevens, A. C., Sloan, M. L., Lullo, C., Visser, S. N., Fox, M. H., Armour, B. S., Campbell, V. A., Brown, D. R., Dorn, J. M., \& Centers For Disease Control And Prevention (CDC) (2014). Vital signs: disability and physical activity--United States, 2009-2012. MMWR. Morbidity and mortality weekly report, 63(18), 407-413.

Chantal Y, Guay F, Dobreva-Martinova T, Vallerand RJ. (1996). Motivation and elite performance: an exploratory investigation with Bulgarian athletes. Int J Sport Psychol. 27(2):173-182.

Cottingham, M., Phillips, D.H., Hall, S.A., Gearity, B.T., \& Carroll, M.S. (2014). Application of the Motivation Scale for Disability Sport Consumption: An Examination of Intended Future Consumption Behavior of Collegiate Wheelchair Basketball Spectators. 37(2): 117-133.

Cronin, L.D., \& Allen, J. (2017). Development and initial validation of the Life Skills Scale for Sport. Psychology of Sport and Exercise, (28): 105-119.

De Hollander, E. L., \& Proper, K. I. (2018). Physical activity levels of adults with various physical disabilities. Preventive medicine reports, 10,370-376.

Deans, S., Burns, D., Mcgarry, A., Murray, K., \& Mutrie, N. (2012). Motivations and barriers to prosthesis users participation in physical activity, exercise and sport: a review of the literature. Prosthetics and orthotics international, 36(3), 260-269.

Devlet İstatistik Enstitüsü (DİE). (2009). 2002 Türkiye Özürlüler Araştırması. Yayın No: 2913. Ankara.

Durstine J.L., Gordon, B., Wang, Z., Luo, X., (2013). Chronic disease and the link to physical activity. J. Sport Health Sci. 2(1):3-11.

Edwards, S. D., Ngcobo H. S. B., Edwards D. J. Ve Palavar K. (2005). Exploring the relationship between physical activity, psychological well-being and physical self-perception in different exercise groups. South African Journal for Research in Sport, Physical Education and Recreation. 27(1):75-90.

Kavussanu, M. (2006). Motivational predictors of prosocial and antisocial behaviour in football. Journal of sports sciences, 24(6), 575-588.

Kingsnorth, S., Healy, H., \& Macarthur, C. (2007). Preparing for adulthood: a systematic review of life skill programs for youth with physical disabilities. The Journal of adolescent health: official publication of the Society for Adolescent Medicine, 41(4), 323-332.

Kosowan, L., Wener, P., Holmqvist, M., Gonzalez, M., Halas, G., Rothney, J., \& Katz, A. (2019). Physical activity promotion in Manitoba: Strengths, needs, and moving forward. SAGE open medicine, 7 ,

Küçük, V. \& Koç, H. Psiko-sosyal gelişim süreci içerisinde insan ve spor ilişkisi. Dumlupınar Üniversitesi Sosyal Bilimler Dergisi, 2004; 10: 131-141.

Lee, O., Park, M., Jang, K., \& Park, Y. (2017). Life lessons after classes: investigating the influence of an afterschool sport program on adolescents' life skills development. International journal of qualitative studies on health and well-being, 12(1): 1307060.

Moreno, J. A. \& Cervelló, E. (2005). Physical self-perception in spanish adolescents: effects of gender and involvent in physical activity. Journal of Human Movement Studies, 48, 291 311.

O'neil, L., \& Hodge, K. (2019). Commitment in Sport: The Role of Coaching Style and Autonomous versus Controlled Motivation, Journal of Applied Sport Psychology, DOI: 10.1080/10413200.2019.1581302

Özdinç, Ö. (2005). Çukurova üniversitesi öğrencilerinin sporun ve spora katılımın sosyalleşmeyle ilişkisi üzerine görüşleri. Spormetre Beden Eğitimi ve Spor Bilimleri Dergisi. 3(2), 77$84.021 /$ asbid. 752630

Özdinç, Ö. Çukurova üniversitesi öğrencilerinin sporun ve spora katılımınsosyalleşmeyle ilişkisi üzerine görüşleri. Spormetre Beden Eğitimi ve Spor Bilimleri Dergisi, 2005; 3 (2): 77-84.

Perkins, D. F., \& Noam, G. G. (2007). Characteristics of sports-based youth development programs. New directions for youth development, (115), 75-9. 
Rogers, H., Morris, T., \& Moore, M. (2008). Rekreasyonel egzersiz katılımcılarının başarı hedeflerine iliş̧in nitel bir çalışma. Qual Rep. 13 (4): 706-734.

RUBIN, S.E., CHAN, F., \& THOMAS, D.L. (2003). Assessing changes in life skills and quality of life resulting from rehabilitation services. Journal of Rehabilitation, 69 (3): 4-9.

Skatteboe, S., Roe, C., Perrin, P. B., Dalen, H., Bautz-Holter, E., Nyquist, A., \& Saebu, M. (2016). One-year trajectories of motivation and physical activity in persons with disabilities. Journal of rehabilitation medicine, 48(4), 371-377.

Tekkurşun D, G., \& Cicioğlu H.İ. (2018). Motivation Scale For Participation in Physical Activity (MSPPA): A study of validity and reliability. International Journal of Human Sciences. 15 (4): 2479-2492.

Top E, Akil M, Aydin N. (2018). The effects of the taekwondo training on children's strengthagility and body coordination levels. Journal of Teaching Research and Media in Kinesiology, 1(1), $1-10$.

Top, E., \& Akil, M. (2018). Effects of a 3-month recreative exercise applied to individuals with intellectual disability on their electromyogram (EMG) variations and balance performance. International Journal of Developmental Disabilities, 64(4-5), 282-287.

Top, E., Kibris, A., \& Kargi, M. (2020). Effects of Turkey's folk dance on the manual and body coordination among children of 6-7 years of age, Research in Dance Education, 21:1, 3442.

Trost Sg, Owen N, Bauman Ae, Sallis Jf, Brown W. (2002). Correlates of adults' participation in physical activity: review and update. Med Sci Sports Exerc, 34: 1996-2001.

Ulukan, M . (2020). Ortaöğretim Öğrencilerinin Fiziksel Aktiviteye Katılım Motivasyonları ve Sürekli Umut Düzeylerinin İncelenmesi . Akdeniz Spor Bilimleri Dergisi , 3 (1), 202-218 . DOI: 10.38

Van Der Ploeg, H. P., Van Der Beek, A. J., Van Der Woude, L. H., \& Van Mechelen, W. (2004). Physical activity for people with a disability: a conceptual model. Sports medicine (Auckland, N.Z.), 34(10), 639-649.

WHO. (2011). World Report In Disability. Publications of the World Health Organization Whyte ve Ingstad. Disability and Culture: An Overview. ISBN: $978 \quad 92 \quad 4 \quad 156418 \quad 2$ (file:///C:/Users/user/Downloads/9789240685215_eng.pdf. Acces: 15.08.2020). WHO Press, Geneva.

Yetim, A., (2005), Sosyoloji ve Spor. Topkar Matbaacıllk, Trabzon, 119 